\title{
Método de calibração simples de um espectrômetro de UV-vis de baixo custo
}

DOI: $10.30609 / J E T I .2018-7523$

\section{Matheus C. S. Ribeiro ${ }^{1}$, Ronaldo C. Viscovini ${ }^{2 *}$}

1 UFMS - Universidade Federal de Mato Grosso do Sul, Campo Grande, Mato Grosso do Sul, Brasil

2UEM - Universidade Estadual de Maringa, Goioere, Paraná, Brasil

\section{Resumo}

Espectrômetros ópticos são instrumentos utilizados para se analisar a interação da radiação eletromagnética com a matéria. Os espectrômetros, na maioria das vezes, utilizam sistemas mecânicos para desviar a trajetória da luz, fazendo com que se varie o comprimento de onda incidente na matéria, ou para os espectrômetros mais modernos pode-se empregar CCDs (charge-coupled devices) como sensores de luz. Neste relato técnico, descrevemos o procedimento de calibração de um espectrômetro óptico com CCD, utilizando o espectro de lâmpadas fluorescentes comerciais como referência. Os resultados obtidos mostraram que o espectrômetro foi devidamente calibrado, e que o processo de calibração pode ser usado para difundir o conhecimento de espectroscopia óptica, tanto para os acadêmicos quanto para os alunos do ensino médio, proporcionando questionamentos ou sanando dúvidas acerca da natureza das cores e do espectro eletromagnético.

Palavras-Chave: Espectroscopia óptica, Espectrômetro, Charge-coupled devices, Calibração. 


\section{1.- Espectrômetro Compacto com CCD acoplado à fibra óptica}

Um espectrômetro óptico é um instrumento utilizado para analisar os componentes espectrais de uma radiação eletromagnética (Luz). Para isso geralmente utiliza elementos dispersores (prismas e grades de difração), que desviam a trajetória da radiação conforme seu comprimento de onda até um sistema de detecção. A Figura 1 ilustra o princípio de funcionamento de um espectrômetro óptico com prisma. Para selecionar os comprimentos de ondas a serem analisados, é empregado um sistema mecânico para mover o elemento dispersor, o detector e/ou espelhos acessórios.

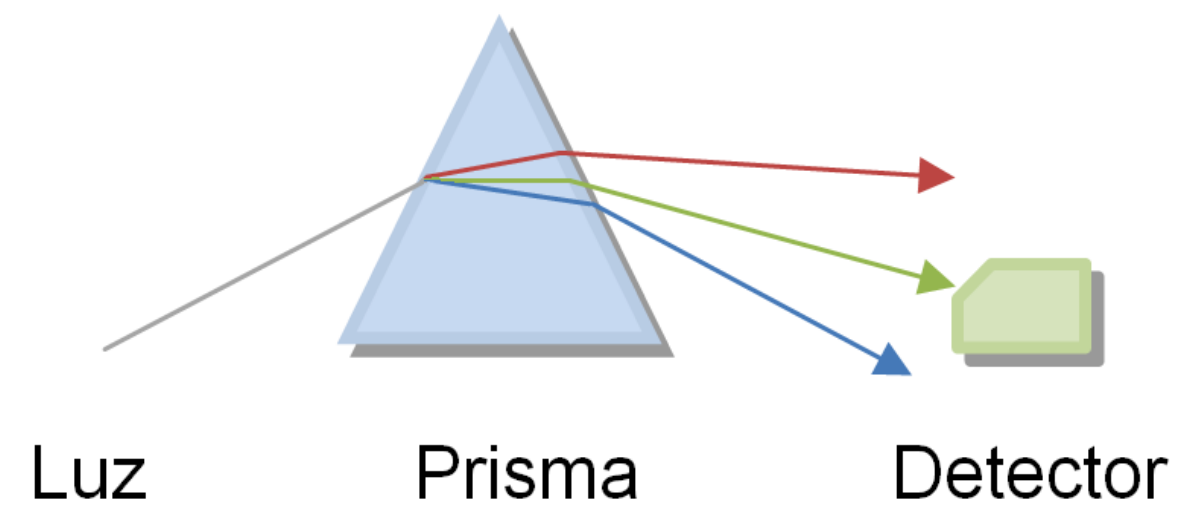

Figura 1 - Esquema ilustrativo dos principais componentes de um espectrômetro com prisma

Com o advento dos charge-coupled devices (CCDs) foi possível compactar os espectrômetros, eliminando a necessidade de sistemas mecânicos, devidos aos CCDs serem constituídos por milhares (ou milhões) de minúsculos sensores ópticos em linha (ou numa superfície). Estes componentes são encontrados usualmente nas máquinas fotográficas e filmadoras digitais, que tem demonstrado seu potencial para o ensino de física [1] ao longo dos anos. Na Figura 2 tem-se a parte óptica de um espectrômetro compacto utilizando CCD com entrada de fibra óptica. 


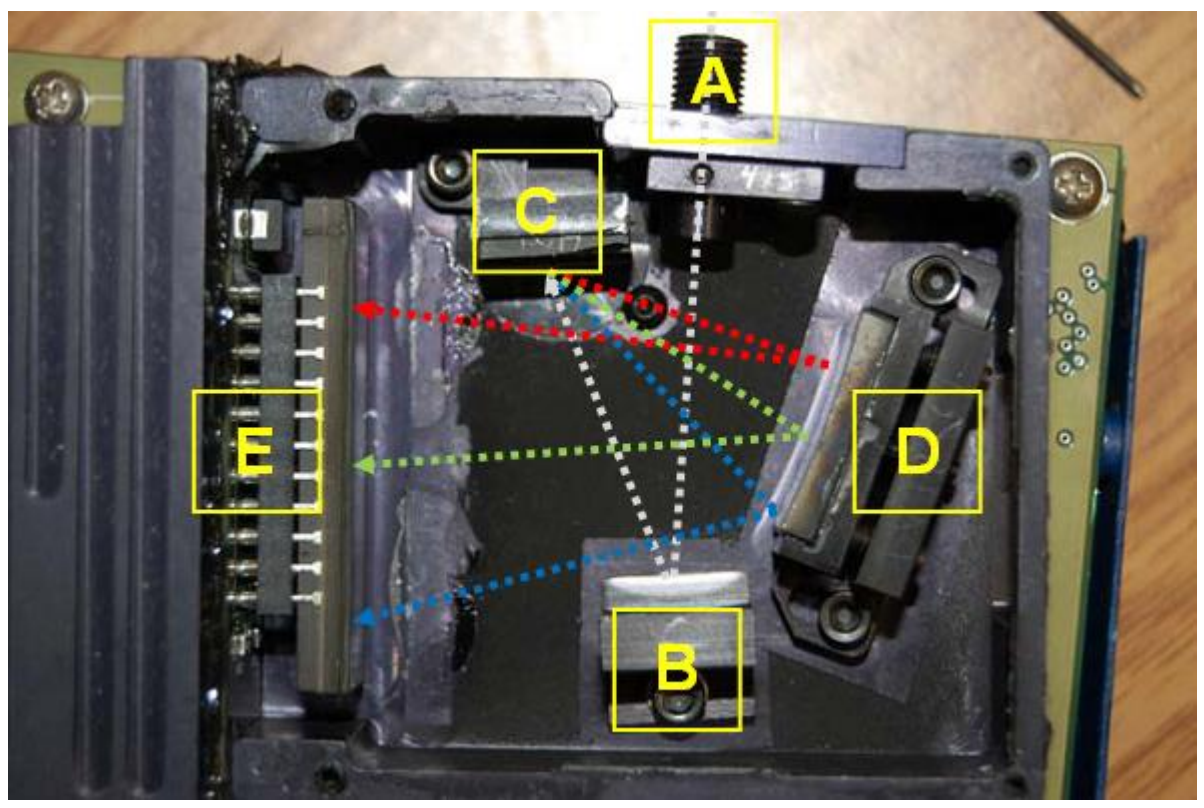

Figura 2 - Espectrômetro Compacto com CCD: (A) conector de entrada para fibra óptica, (B) espelho de colimação, (C) grade de difração, (D) espelho de focalização e (E) Detector CCD.

Existem diversos modelos de espectrômetros compactos com CCD no mercado. Dependendo do fabricante, qualidade da óptica (espelhos e rede de difração) e da resolução e sensibilidade da CDD, um equipamento deste tipo pode chegar a custar muitos milhares de dólares americanos. A Figura 3 ilustra o equipamento utilizado neste trabalho, disponível no mercado Norte Americano por duas centenas de dólares e fabricado pela empresa Science-Surplus.

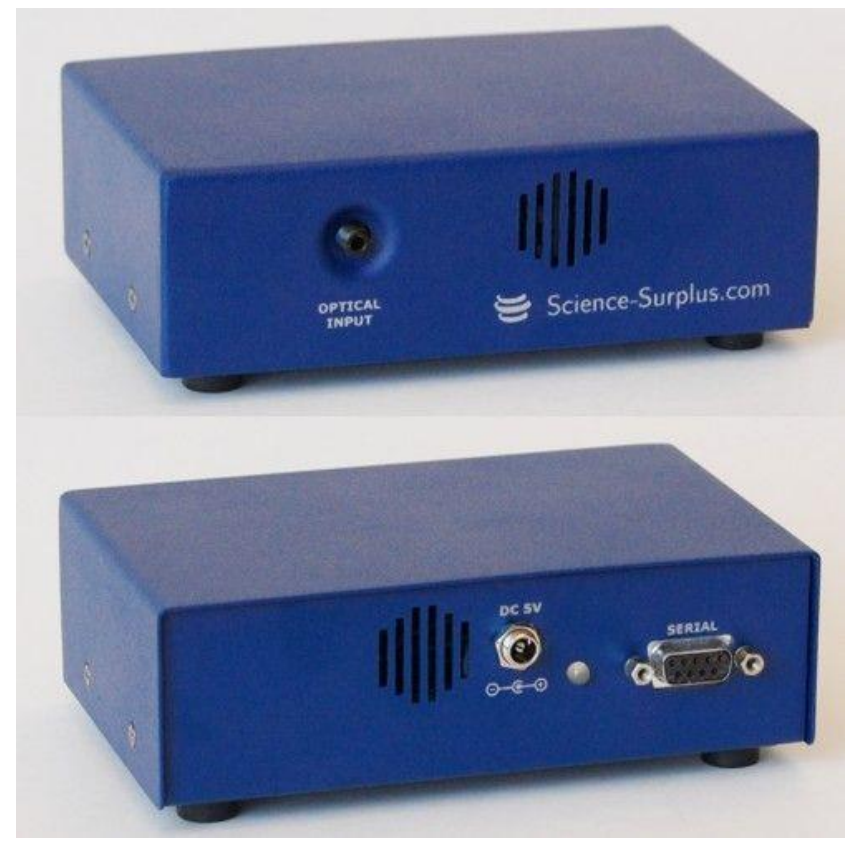

Figura 3 - Imagem de frente e verso do espectrômetro compacto de CCD da ScienceSurplus. 
Esse espectrômetro utiliza uma CCD Sony ILX511 com 2048 detectores ópticos numa linha, digitalização de 16 bits, tempo de leitura de $350 \mathrm{~ms}$ e, dependendo da grade de difração, permite analisar boa parte da luz visível, com resolução típica de $1 \mathrm{~nm}$, disposto em uma caixa compacta de dimensões $15 \mathrm{~cm}$ x $10 \mathrm{~cm}$ x $5 \mathrm{~cm}$. A comunicação com microcomputador é realizada através de uma interface serial (RS232C), o software Spectrum Studio-disponibilizado gratuitamente com o espectrômetro, compatível com o Windows ${ }^{\circledR}$, permite visualizar, gravar, integrar e calcular médias de espectros.

\section{2.- Calibração do Espectrômetro de CCD}

O espectrômetro utilizado neste trabalho é fornecido sem calibração prévia pela empresa fabricante. Um espectrômetro não calibrado capta o espectro luminoso, fornecendo a natureza espectral da luz analisada, entretanto o intervalo de comprimento de onda se encontra deslocado e necessita ser ajustado para valores corretos. A aquisição de um espectrômetro já calibrado de fabrica aumenta consideravelmente seu custo. 0 processo de calibração pelo operador, deste espectrômetro em particular, representa uma excelente oportunidade para compreensão e/ou aprendizagem de conceitos básicos de óptica.

A calibração consiste em associar a cada um dos 2048 sensores o comprimento de onda real do espectro detectado. Para evitar o árduo trabalho de montar uma tabela com 2048 valores de conversão, é possível fazer uma boa aproximação através de uma função de conversão $\lambda(n)$, na qual essa função fornece o comprimento de onda (em $\mathrm{nm}$ ), para cada sensor óptico numerado (n) de 0 a 2047. A função $\lambda(n)$, obtida através da Equação 1, será um polinômio de terceiro grau, truncada de uma série de Taylor centrada em $n=0$ (série de Maclaurin):

$$
\lambda(n)=C_{0}+C_{1} n+C_{2} n^{2}+C_{3} n^{3}
$$

O software Spectrum Studio é apto a realizar as conversões apropriadas, de modo que a calibração consista apenas em informar os valores dos coeficientes $C_{0}, C_{1}, C_{2}$ e $C_{3}$.

Para determinar os valores dos coeficientes é necessário conhecer os comprimentos de ondas de um espectro padrão como referência. Existem fontes luminosas específicas para esta função, que podem ser muito dispendiosas. Neste trabalho utilizou-se como referência uma simples lâmpada fluorescente compacta, disponível 
comercialmente, que emite um espectro característico, conforme ilustrado na Figura 4. Podem-se visualizar quatro picos principais sendo dois característicos do vapor de mercúrio [2] ( $\lambda_{A}=436 \mathrm{~nm}$ e $\left.\lambda_{C}=546 \mathrm{~nm}\right)$ e dois devido à fluorescência do material que reveste a lâmpada $\left(\lambda_{B}=488 \mathrm{~nm}\right.$ e $\left.\lambda_{D}=612 \mathrm{~nm}\right)$.

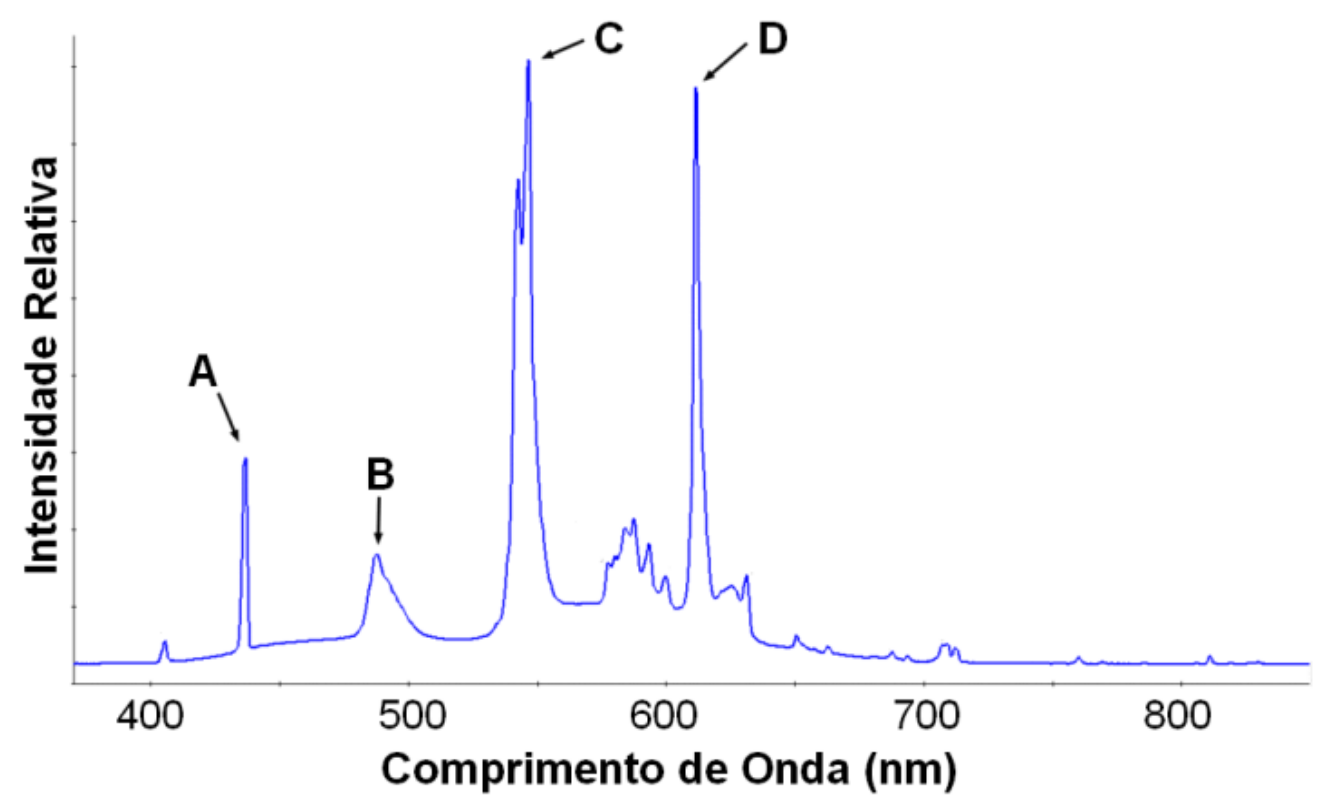

Figura 4 - Espectro característico de uma lâmpada fluorescente com seus picos de emissão: A) $\lambda_{A}=436 \mathrm{~nm}$, B) $\lambda_{B}=488 \mathrm{~nm}$, C) $\lambda_{C}=546 \mathrm{~nm}$ e D) $\lambda_{D}=612 \mathrm{~nm}$.

Na primeira etapa da calibração os coeficientes no Spectrum Studio são ajustados para os valores iniciais: $C_{0}=0, C_{1}=1, C_{2}=0$ e $C_{3}=0$. Isto faz com que a escala corresponda diretamente o número do sensor, ou seja: $\lambda(n)=n$.

\section{3.- Resultados Finais}

A Figura 5 ilustra um espectro não calibrado de uma lâmpada fluorescente compacta Osram ${ }^{\circledR}$ modelo $827,15 \mathrm{~W}, 2700 \mathrm{~K}$, colhido nas condições anteriores. 


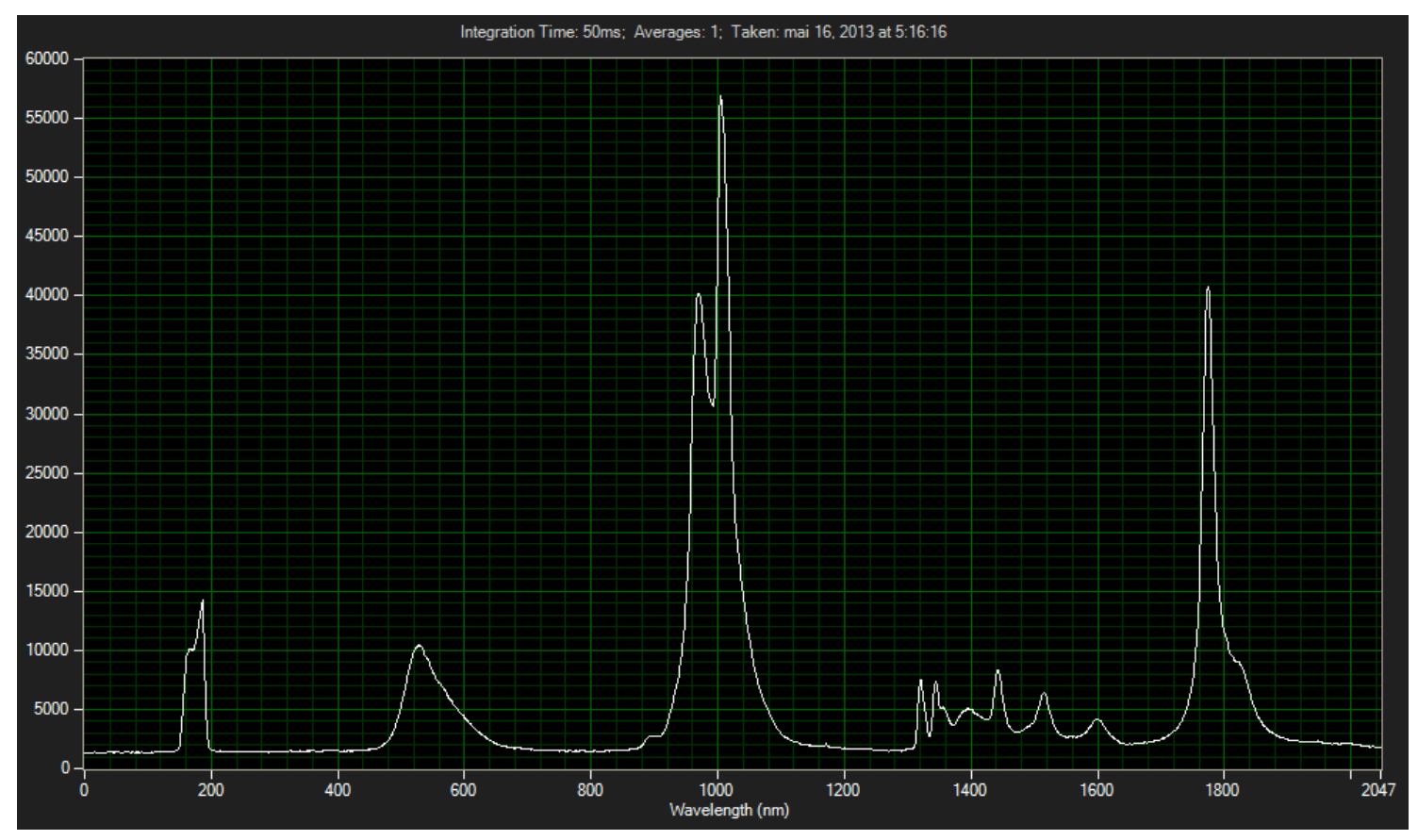

Figura 5 - Espectro de uma lâmpada fluorescente comercial sem calibração.

Observando o gráfico e analisando o arquivo de valores criados pelo software, encontra-se o número dos sensores (n) correspondentes aos quatro picos de emissão $(\lambda)$, que no exemplo foram: para $\lambda_{A}=436 \mathrm{~nm}, n_{A}=186 ; \lambda_{B}=488 \mathrm{~nm}, n_{B}=528 ; \lambda_{C}=546 \mathrm{~nm}, n_{C}=$ 1005; $\lambda_{D}=612 \mathrm{~nm}, n_{D}=1774$. Estes quatro conjuntos de valores de $\lambda$ e $n$ aplicados na $\lambda(n)$ geram um sistema que quatro equações com quatro incógnitas $\left(C_{0}, C_{1}, C_{2}\right.$ e $\left.C_{3}\right)$. Resolvendo o sistema encontram-se os valores dos coeficientes, que no exemplo foram: $C_{0}=403,5 ; C_{1}=$ $0,183, C_{2}=-4,64 \times 10^{-5}$ eC3 $=5,34 \times 10^{-9}$. Atualizando o software com os valores dos coeficientes encontrados, tem-se o espectro calibrado de uma lâmpada fluorescente, conforme ilustrado na Figura 6. Nota-se que esse espectrômetro tem uma faixa de detecção entre 403,5nm e 629,48nm, ou seja, boa parte da região visível, com exceção do extremo do espectro correspondente ao vermelho (630-700nm). 


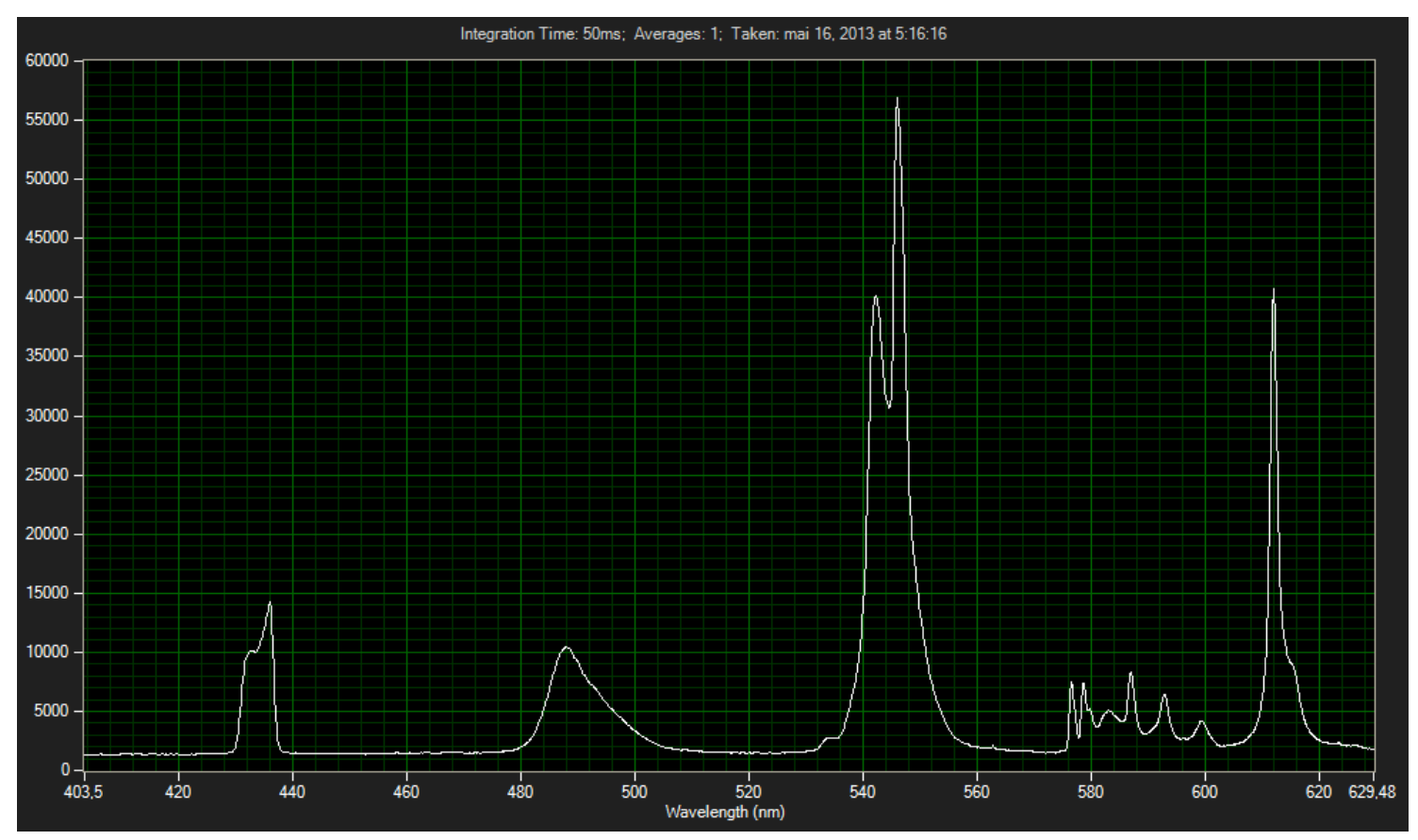

Figura 6 - Espectro calibrado de uma lâmpada fluorescente comercial

Assim, dispomos de um espectro calibrado e de baixo custo que pode ser empregado para diversos usos, seja em ensino ou pesquisa [1-4].

\section{4.- Comentários Finais}

Este espectrômetro de baixo custo pode ser empregado para o estudo didático de espectros emitido por lâmpadas comerciais, ou ainda empregado em diversos experimentos de interesse. A relação custo benefício em se obter um espectro e poder calibrar o mesmo de acordo com a necessidade torna o aparato ainda mais interessante.

\section{5.- Bibliografia}

[1] O.C.N. PEREIRA, W.M. SILVA, A.C. SABINO, M.E. GOZZI, A.R. SAMPAIO, R.C. VISCOVINI, Software de efeito estroboscópico por superposição de frames de videoclipes aplicada no ensino de Cinemática. Caderno Brasileiro de Ensino de Física, v.29, n.2, 2012. 
[2] R.R.G. PARANHOS, V.L. RICHARD, P.S. PIZANI. Lâmpada de Hg para experimentos e demonstrações de física moderna: introdução ao efeito fotoelétrico e outros tópicos. Revista Brasileira de Física, v.30, n.4, 2008.

[3] T.C. ZANETTI, J.S. CABRAL. Calculation of an optical steup for a LIBS system. Journal of Experimental Techniques and Instrumentation, v.1, n.1, 2018.

[4] T.A. CANASSA, A.L. LAMONATO, A.V. RIBEIRO. Utilização da lei de Lambert-Beer para determinação da concentração de soluções. Journal of Experimental Techniques and Instrumentation, v.1, n.2, 2018. 